\title{
Enfermedad de Kienböck: conceptos actuales
}

\author{
Pablo De Carli, Ezequiel Zaidenberg \\ Sector de Cirugía de Mano y Reconstructiva de Miembro Superior, Instituto de Ortopedia y Traumatología \\ "Prof. Dr. Carlos E. Ottolenghi", Hospital Italiano de Buenos Aires, Ciudad Autónoma de Buenos Aires, Argentina
}

\section{RESUMEN}

La osteonecrosis del semilunar o enfermedad de Kienböck es un cuadro progresivo y debilitante que puede llevar al dolor crónico y la pérdida de la función. Pese a que se conoce hace más de 100 años, la etiología todavía es incierta, si bien se la ha relacionado con factores mecánicos, vasculares y traumáticos. Su evolución natural está pobremente definida y los hallazgos radiográficos no siempre se correlacionan con los hallazgos clínicos. Se han logrado avances para identificar y entender la progresión del proceso avascular y sus efectos nocivos sobre la mecánica de la muñeca. El tratamiento inicial es no quirúrgico. El perfeccionamiento de la técnica quirúrgica utilizando injertos óseos vascularizados de radio distal puede mejorar los resultados en los pacientes que cursan etapas tempranas de la enfermedad, aunque resta mucho trabajo para determinar si este tratamiento quirúrgico representa una mejor opción que los tratamientos tradicionales. Reportes recientes de resultados a largo plazo de la osteotomía de acortamiento del radio para estadios tempranos de la enfermedad y la resección de la primera fila del carpo para estadios avanzados revelan que estos procedimientos son una opción válida para el tratamiento a largo plazo de este cuadro de difícil manejo. Palabras clave: Enfermedad de Kienböck.

\section{Kienböck's disease: current concepts}

\section{ABSTRACT}

Osteonecrosis of the lunate or Kienböck's disease is a progressive, debilitating condition that can lead to chronic pain and function loss. Despite it was identified almost 100 years ago, the etiology remains unknown, although mechanical, vascular, and traumatic factors have been associated. The natural history of Kienböck's disease is poorly defined, and the radiographic findings do not always correlate with the clinical findings. There has been some progress in identifying and understanding the progression of the avascular process and its deleterious effects on wrist mechanics. Initial treatment is conservative. Improvement in surgical techniques with vascularized bone grafts from the distal radius may lead to an outcome improvement for patients in the early stages of disease. However, more research is still needed to determine whether this surgical treatment represents an improvement over conventional treatment alternatives. Recent reports of long-term outcomes for radial shortening osteotomy in osteonecrosis early stage patients and for proximal row carpectomy (PRC) in advanced Kienböck's disease patients reveal that these procedures provide reliable options for the long-term management of this challenging condition.

Key words: Kienböck's disease.

\section{INTRODUCCIÓN}

La osteonecrosis del semilunar o enfermedad de Kienböck es un cuadro progresivo y debilitante que puede llevar al dolor crónico y la pérdida de la función. Pese a que esta enfermedad se conoce hace más de 100 años, su etiología todavía es incierta, aunque se la ha relacionado con factores mecánicos, vasculares y traumáticos. Su evolución natural está pobremente definida y los hallazgos radiográficos no siempre se correlacionan con los hallazgos clínicos. Se han logrado avances en la identificación y el entendimiento de la progresión del proceso avascular y sus efectos nocivos sobre la mecánica de la muñeca. El tratamiento inicial es no quirúrgico. El perfeccionamiento

Recibido el 30-7-2020. Aceptado luego de la evaluación el 31-7-2020 • Dr. PABLO DE CARLI • pablo.decarli@ gmail.com Cómo citar este artículo: De Carli P, Zaidenberg E. Enfermedad de Kienböck: conceptos actuales. Rev Asoc Argent Ortop Traumatol 2020;85(Supl.):S26-S35. https://doi.org/10.15417/ issn. 1852-7434.2020.85.4S.1170 
de la técnica quirúrgica utilizando injertos óseos vascularizados de radio distal puede mejorar los resultados en los pacientes que cursan etapas tempranas de la enfermedad, aunque resta mucho trabajo para determinar si este tratamiento quirúrgico representa una mejor opción que los tratamientos tradicionales. Reportes recientes de resultados a largo plazo de la osteotomía de acortamiento del radio para estadios tempranos de la enfermedad y la resección de la primera fila del carpo para estadios avanzados revelan que estos procedimientos son una opción válida para el tratamiento a largo plazo de este problema de difícil manejo.

\section{PERSPECTIVA HISTÓRICA}

Robert Kienböck, radiólogo austríaco, realizó la primera descripción de la necrosis avascular del semilunar. ${ }^{1}$ Su trabajo "Concerning traumatic malacia of the lunate and its consequences" "Traumatismo del semilunar y sus consecuencias") fue publicado en 1910. En este artículo, Kienböck describió una enfermedad aislada del semilunar asociada a cambios secundarios en otros huesos del carpo. Los pacientes tenían dolor, pérdida de la movilidad y una prominencia en el área de la muñeca. La evaluación radiográfica reveló cambios aislados en la región proximal del semilunar, con eventual colapso y fragmentación. Kienböck adjudicó este proceso a una "alteración en la nutrición" del semilunar secundaria a un trauma repetitivo.

En 1928, Hultén, al evaluar a 23 pacientes de origen sueco con enfermedad de Kienböck, notó que el 78\% poseía una varianza cubital negativa comparado con el $23 \%$ que presenta la población en general. ${ }^{2}$ Este estudio condujo a creer que había una asociación entre varianza cubital negativa y necrosis avascular del semilunar, y generó grupos que defendían la etiología mecánica de la enfermedad. Estudios más recientes que evaluaron a pacientes de etnia asiática demostraron que la enfermedad de Kienböck puede ocurrir en pacientes con varianza cubital neutra o positiva.

\section{ETIOLOGÍA}

El semilunar es el hueso central de la primera fila del carpo y articula proximalmente con la fosa semilunar del radio y el complejo del fibrocartílago triangular y distalmente con los huesos grande y ganchoso. El semilunar y la fosa semilunar del radio tienen una forma hemisférica y un alto grado de congruencia entre las superficies articulares. Se cree que este factor anatómico desempeña un papel importante en el hecho de que la articulación entre el radio y el semilunar es una de las últimas en desarrollar cambios degenerativos.

Dado que la enfermedad de Kienböck es fundamentalmente un proceso avascular, los patrones de vascularización del hueso semilunar han recibido mucha atención para intentar esclarecer el desarrollo de la osteonecrosis. ${ }^{4,5}$ Se han identificado múltiples patrones de vascularización en muestras cadavéricas, pero la mayoría se nutre de vasos arteriales dorsales y palmares. ${ }^{5}$ Estos vasos sanguíneos ingresan en el hueso a través de sus superficies no articulares. En un estudio, el 7-23\% de los semilunares estaban irrigados a través de una única arteria dorsal. ${ }^{4,5}$

Cuando el vaso o los vasos ingresan en el hueso, se pueden encontrar varios patrones de ramificación intraósea, incluidos los patrones "I", "X" e "Y". Se ha postulado que los semilunares que poseen un único aporte arterial o aquellos con patrones de ramificación intraósea limitados pueden ser más proclives a desarrollar osteonecrosis secundaria a un trauma agudo o crónico repetitivo. La presencia de un suministro vascular palmar explica por qué pacientes con inestabilidad y luxación perilunar, rara vez, desarrollan cambios avasculares en el semilunar, ya que las uniones capsulares volares al semilunar permanecen intactas. ${ }^{6}$

La interrupción del flujo venoso que conduce a un aumento de la presión intraósea también podría desencadenar cambios avasculares y osteonecrosis. Las presiones intraóseas de semilunares necróticos halladas in vitro estaban aumentadas en comparación con la presión de semilunares normales. En un estudio, se examinó el drenaje venoso en especímenes cadavéricos y se halló un plexo denso de pequeños vasos venosos en la superficie perióstica de las caras palmar y dorsal de los huesos. ${ }^{7}$ Los autores afirman que el plexo podría ser un sitio de interrupción del flujo venoso, secundario a factores de compresión locales y sistémicos.

Asimismo, se ha evaluado la contribución de los factores mecánicos en el desarrollo de la osteonecrosis ${ }^{8.9}$ Como ya se mencionó, durante mucho tiempo, a la relación entre la altura del radio y el cúbito en las articulaciones radiocubital distal y radiocarpiana (varianza cubital) se le ha adjudicado un papel en el desarrollo de esta condición. Otros factores mecánicos, como la inclinación radial, podrían contribuir a la aparición de este cuadro, particularmente en aquellos pacientes con varianza cubital neutra o positiva en quienes el aumento de las tensiones radiosemilunares secundarias a la varianza cubital negativa no generaría el proceso avascular. Se ha identificado que los pacientes con inclinación radial disminuida tienden a presentar semilunares más pequeños y una asociación con la enfermedad de Kienböck. ${ }^{8}$ 
Ciertos factores sistémicos también se han asociado con la osteonecrosis del semilunar. Una tendencia a la hipercoagulabilidad o a la congestión venosa, o la disminución del flujo de entrada arterial pueden desempeñar un papel en este proceso. ${ }^{6} \mathrm{El}$ uso de corticoides sistémicos, la enfermedad de células falciformes, la parálisis cerebral y la embolia séptica se han asociado con osteonecrosis, aunque no existe una correlación definida entre estas afecciones sistémicas y la osteonecrosis del semilunar. A estas alturas, luego de 100 años desde el trabajo de Kienböck, la etiología permanece indefinida. Aunque la aparición de esta enfermedad se ha atribuido a orígenes mecánico, anatómico y sistémico, no se ha identificado ningún mecanismo etiológico específico. Es muy probable que una compleja interrelación entre todos los factores descritos contribuya al proceso de la enfermedad. ${ }^{6,8,9}$

\section{HALLAZGOS CLÍNICOS Y RADIOGRÁFICOS}

Los pacientes con enfermedad de Kienböck suelen presentarse con dolor y debilidad en la muñeca, a menudo, sin antecedentes de trauma agudo. Pueden informar que han sufrido síntomas durante un tiempo variable antes de la consulta, aunque, con frecuencia, referirán un período de larga evolución de síntomas progresivos. Por esta razón, la evolución natural de esta afección no se conoce bien. El dolor puede variar de leve y ocasional a severo y debilitante. Es más común en hombres adultos jóvenes (de 20 a 40 años) y es rara en niños, aunque algunos informes han documentado casos en este grupo etario. Rara vez es bilateral.

En el examen físico, se observa una tumefacción dolorosa a la palpación en la región dorsal de la muñeca en relación con el semilunar, probablemente debida a sinovitis. Casi siempre el rango de movilidad está disminuido, con pérdida de la flexión y de la extensión. Típicamente hay dolor en los extremos del rango de movilidad, sobre todo, en extensión. La rotación del antebrazo suele estar conservada. También la fuerza de agarre es considerablemente menor comparada con la de la muñeca contralateral.

Los estudios radiográficos son diagnósticos para la enfermedad de Kienböck. Según el estadio, las radiografías pueden mostrar esclerosis difusa del semilunar, cambios quísticos, fragmentación, colapso de la superficie articular y cambios artríticos perilunares. A menudo, se puede visualizar una fractura en la incidencia lateral durante las primeras etapas de la enfermedad, y no está claro si esto representa una lesión real o si la línea de fractura es el resultado de la disminución de las propiedades estructurales del hueso necrótico. Las imágenes radiográficas también son importantes para determinar las propiedades anatómicas y mecánicas asociadas de la muñeca involucrada, como varianza cubital, inclinación radial, altura del carpo, ángulo radioescafoideo y el tamaño del semilunar.

La resonancia magnética es un importante estudio diagnóstico, particularmente útil en las etapas tempranas de la enfermedad, cuando los hallazgos radiográficos pueden ser nulos. En las imágenes en la secuencia T1, la pérdida de la grasa de la médula ósea provoca una disminución de la intensidad de la señal. De la misma manera, las imágenes en la secuencia T2 también revelan una baja intensidad de señal. Para establecer el diagnóstico de enfermedad de Kienböck, es imperativo que los cambios de señal sean difusos y afecten a la totalidad del semilunar. Cuando los cambios en la intensidad de la señal se localizan en una zona determinada del hueso, se deben sospechar otros diagnósticos. Por ejemplo, si los cambios se limitan a la región proximal cubital del semilunar, se debe considerar una impactación cúbito-carpiana. Otros estudios diagnósticos también pueden ser de ayuda. Antes de la resonancia magnética, la gammagrafía ósea era útil como estudio diagnóstico si las radiografías eran negativas. Finalmente, la tomografía computarizada puede servir para evaluar el colapso de la superficie articular y la presencia de fracturas.

Cuando las radiografías simples no revelan signos obvios de compromiso de la articulación radiocarpiana o mediocarpiana, la artroscopia diagnóstica también puede ser útil para establecer el estadio de la enfermedad. Bain y Begg han descrito una clasificación artroscópica basada en el número de superficies articulares involucradas. ${ }^{10}$ El sistema clasifica a las superficies articulares semilunares (semilunar proximal, semilunar distal, faceta semilunar del radio) como funcional o no funcional, y se proporciona un algoritmo de tratamiento de acuerdo con los hallazgos artroscópicos. La artroscopia, en este entorno, debe usarse con moderación, ya que las radiografías simples y otros estudios por imágenes son casi siempre diagnósticos en términos de la evaluación de las superficies articulares.

\section{ESTADIOS}

La estadificación de la enfermedad de Kienböck depende principalmente de los hallazgos radiográficos y es una parte crítica de la evaluación y el manejo de los pacientes que sufren este cuadro, ya que define las diferentes opciones terapéuticas posibles en cada caso. Se han sugerido varias clasificaciones, pero el esquema descrito por Lichtman y cols. es el más utilizado y su reproducibilidad y confiabilidad son buenas. ${ }^{6}$ 
En el estadio 1, las radiografías simples revelan tanto densidad como superficies articulares normales del semilunar, pero la resonancia magnética muestra cambios de intensidad de señal difusos. En el estadio 2, el semilunar aparece difusamente esclerótico en la radiografía simple, puede haber líneas de fractura, aunque las superficies articulares están conservadas. El estadio 3 es la etapa más común de presentación inicial y se define por el colapso de la superficie articular del semilunar. Este estadio está subdividido en dos etapas. La etapa 3A se caracteriza por el colapso articular con mantenimiento de la altura y la alineación normal del carpo. La etapa 3B, por el contrario, se define por la presencia de colapso articular del semilunar y rotación secundaria del escafoides, migración proximal del hueso grande y disminución de la altura del carpo. En los últimos años, Lichtman agregó un nuevo estadio, el 3C, caracterizado por los mismos hallazgos que en el estadio 3A, pero con el semilunar dividido, en la radiografía lateral, en dos fragmentos triangulares enfrentados, debido a la fractura en el plano coronal. Por último, la etapa 4 se define por el colapso semilunar asociado con artrosis radiocarpiana o mediocarpiana (Tabla 1).

Tabla 1. Clasificación de Lichtman para la enfermedad de Kienböck

\begin{tabular}{|l|l|}
\hline Estadio1 & Radiografías normales, cambios de intensidad de la señal en la resonancia magnética \\
\hline Estadio 2 & Esclerosis del semilunar en las radiografías; puede haber líneas de fractura \\
\hline Estadio 3 & Colapso de la superficie articular del semilunar \\
\hline Estadio 3A & Alineación y altura normales del carpo \\
\hline Estadio 3B & Rotación del escafoides, migración proximal del hueso grande y pérdida de la altura del carpo \\
\hline Estadio 3C & Fractura en el plano coronal, con el semilunar dividido en un fragmento triangular palmar y otro dorsal \\
\hline Estadio 4 & Colapso semilunar junto con artrosis radiocarpiana o mediocarpiana \\
\hline
\end{tabular}

En un estudio que evaluó la fiabilidad de la clasificación de Lichtman, Goldfarb y cols. hallaron que esta clasificación tiene una considerable fiabilidad interobservador. ${ }^{11}$ Sin embargo, los autores también encontraron que las diferencias identificadas entre las etapas 3A y 3B fueron menos confiables. Por esta razón, propusieron una nueva modificación del sistema de clasificación en el que la etapa 3B se definió como aquella en la que el ángulo radioescafoideo era $>60^{\circ}$. Con la modificación del sistema de estadificación, la fiabilidad interobservador aumentó tanto para la clasificación general (de kappa 0,63 a 0,81), como para la etapa $3 \mathrm{~A}$ (de kappa 0,38 a 0,75). Como resultado, los autores concluyeron en que el ángulo radioescafoideo aumenta la fiabilidad interobservador de la clasificación de Lichtman y la capacidad de distinguir, de manera más confiable, las etapas 3A y 3B de la enfermedad.

\section{TRATAMIENTO}

El tratamiento para la enfermedad de Kienböck está principalmente guiado por la sintomatología y el déficit funcional del paciente, así como por el estadio, como se describió antes. La mayoría de los estudios longitudinales que evalúan el manejo conservador de la enfermedad de Kienböck han identificado la progresión radiográfica de la enfermedad a través de las etapas antes descritas. ${ }^{12-14}$ Keith y cols. ${ }^{14}$ evaluaron a 33 pacientes tratados de manera conservadora y detectaron una pérdida del rango de movilidad (flexión) y una disminución en el estado funcional según los puntajes más bajos del cuestionario DASH. Aunque la evaluación radiográfica longitudinal fue limitada para su serie de pacientes, los autores concluyeron en que la osteonecrosis del semilunar es una enfermedad progresiva.

Hay controversia sobre si la cirugía obtiene mejores resultados que el tratamiento conservador. Delaere y cols. compararon el uso de una férula nocturna con la cirugía en pacientes con enfermedad hasta estadio $3 .{ }^{15}$ La inmovilización produjo resultados equivalentes a los de la cirugía. Estos resultados deben considerarse cuidadosamente, pues, en promedio, la enfermedad del grupo con inmovilización estaba en una etapa más temprana que en el grupo quirúrgico. Otros estudios indican que la cirugía es mejor que el manejo conservador. Salmon y cols. proporcionaron evidencia de que la osteotomía de acortamiento del radio puede ser superior al manejo conservador. 
A pesar de la progresión de la enfermedad, muchos pacientes permanecen funcionales y tienen síntomas tolerables, incluso en estadios avanzados. Por lo tanto, se podría justificar un intento de tratamiento conservador con férula o yeso y medicación analgésica o antinflamatoria en todos los pacientes, al margen del estadio en el momento de la presentación. La inmovilización se debe mantener por tres meses. ${ }^{6} \mathrm{La}$ intervención quirúrgica debe reservarse para los pacientes en los que fracasa el tratamiento conservador y continúan con síntomas.

Existen múltiples procedimientos quirúrgicos para tratar la osteonecrosis del semilunar, y no se ha encontrado demasiada evidencia publicada para determinar qué opciones quirúrgicas son mejores que otras. Pese a las múltiples opciones quirúrgicas disponibles, no hay ninguna que sea superior a otra. Utilizando como guía el esquema de estadios ya descrito, se indican diferentes procedimientos para los distintos estadios. Las variables anatómicas, como varianza cubital e inclinación radial, juegan un papel importante al determinar qué procedimiento es el indicado para cada paciente (Tabla 2).

Tabla 2. Tratamiento de la enfermedad de Kienböck según con el estadio

\begin{tabular}{|c|c|}
\hline Estadio 1 & Inmovilización con yeso por 3 meses \\
\hline $\begin{array}{l}\text { Estadios de } 2 \text { a } 3 \mathrm{~A} \text {, } \\
\text { varianza cubital negativa }\end{array}$ & Acortamiento del radio, alargamiento del cúbito, acortamiento del grande \\
\hline $\begin{array}{l}\text { Estadios de } 2 \text { a } 3 \mathrm{~A}, \\
\text { varianza cubital positiva }\end{array}$ & $\begin{array}{l}\text { Injerto óseo vascularizado y fijador externo, osteotomía de radio en cuña o domo, } \\
\text { acortamiento del grande }\end{array}$ \\
\hline Estadio 3B & $\begin{array}{l}\text { Artrodesis intercarpiana, resección del semilunar, acortamiento del radio, resección de la } \\
\text { primera fila del carpo }\end{array}$ \\
\hline Estadio 4 & Resección de la primera fila del carpo, artrodesis de muñeca, desnervación de la muñeca \\
\hline
\end{tabular}

\section{Estadio 1}

Como en todos los casos, la inmovilización con férula o yeso es la primera opción terapéutica. Se ha utilizado la fijación externa tanto como dispositivo de inmovilización como para descargar el semilunar, aunque la experiencia con esta técnica es limitada. ${ }^{16} \mathrm{Si}$ bien algunos pacientes mejoran con el manejo conservador, se cree que muchos progresan a la etapa 2 , pese al tratamiento. ${ }^{6}$

\section{Estadios 2 y $3 \mathrm{~A}$}

Estas etapas generalmente se consideran juntas respecto a las opciones terapéuticas, cuando el objetivo principal es revascularizar el semilunar para evitar la progresión de la enfermedad. La única excepción de esta agrupación son los procedimientos de revascularización directa, utilizados principalmente para pacientes en estadio 2 cuando el colapso articular aún no ha ocurrido. Sin embargo, muchos autores han recurrido a estos procedimientos cuando hay algún colapso con pérdida de la altura del semilunar, en lo que se ha denominado etapa $3 \mathrm{~A}$ temprana.

Se han descrito varios tipos de procedimientos de revascularización para tratar la enfermedad en estadio $2,{ }^{17-22}$ que incluyen injertos vascularizados del pisiforme, injertos pediculados vascularizados del radio distal (incluye el pronador cuadrado), implantación directa de arterias metacarpianas e injertos vascularizados libres, entre otros. Además de los injertos vascularizados, comúnmente se asocia algún procedimiento de descarga para disminuir las tensiones mecánicas en el semilunar (fijación externa, fijación de la articulación escafotrapeciotrapezoidal o escafogrande). ${ }^{20,21}$

En los últimos tiempos, se ha prestado más interés a los injertos óseos vascularizados del radio distal. ${ }^{19}$ Se han descrito varios tipos de injertos sobre la base de su ubicación con respecto a los compartimentos extensores de la muñeca y también en función de si la arteria donante está ubicada fuera o dentro del compartimento extensor. El injerto más común para tratar la enfermedad de Kienböck está basado en la arteria extracompartimental 4-5. El flujo sanguíneo retrógrado desde la arteria extracompartimental 5 . $^{\mathrm{a}}$ se dirige en dirección anterógrada hacia la arteria extracompartimental $4 .{ }^{a}$ mediante la ligadura de la rama posterior de la arteria interósea anterior. Este injerto presenta un pedículo largo y de gran diámetro, de ubicación cubital. Moran y cols. evaluaron a 26 pacientes con un seguimiento promedio de 31 meses. ${ }^{22}$ En la mayoría, el dolor se alivió y la fuerza de prensión mejoró. El $71 \%$ de 
este grupo tuvo evidencia de revascularización del semilunar. A pesar del éxito general del procedimiento, el 23\% de los pacientes mostró evidencia radiográfica de progresión de la enfermedad en el último seguimiento.

Se realizan injertos óseos vascularizados de pisiforme tomando como sitio donante el hueso pisiforme homolateral, junto con su suministro de sangre, desde pequeñas ramas dorsales y radiales de la arteria cubital. El injerto óseo pediculado es entonces insertado en un defecto óseo creado quirúrgicamente en el dorso de la superficie del semilunar. Daecke y cols. describieron su experiencia en 23 pacientes con estadios 2 o 3A tratados mediante este procedimiento y un seguimiento promedio de 12 años. ${ }^{18} \mathrm{El}$ dolor y la función mejoraron en 20 de 23 pacientes. La enfermedad progresó en seis $(33 \%)$ de los 20 pacientes que contaban con radiografías preoperatorias.

Asimismo, se han utilizado injertos vascularizados libres para tratar la osteonecrosis del semilunar. Gabl y cols. evaluaron a 18 pacientes con enfermedad en estadio 3 tratados con implantación de injerto óseo corticoesponjoso ilíaco vascularizado libre. ${ }^{23}$ La muñeca se estabilizó con un fijador externo durante la curación. Con un seguimiento medio de 5 años, el injerto se incorporó al semilunar en 16 pacientes y no ocurrió colapso carpiano en estos casos. En los otros dos pacientes, el injerto no se integró y fue reabsorbido. En un estudio de seguimiento, Arora y cols. descubrieron que, a los 10 años de seguimiento, los 16 pacientes todavía no tenían evidencia de progresión de la enfermedad o colapso carpiano. ${ }^{24}$

Otras opciones para pacientes en estos estadios incluyen los procedimientos de "nivelación articular" ${ }^{6,8,9} \mathrm{Se}$ cree que descargan mecánicamente el semilunar, lo que lleva a la revascularización y a prevenir la progresión del colapso de la superficie articular. La determinación de qué procedimiento es el adecuado para cada paciente depende principalmente de la relación de longitud entre el radio y el cúbito en la muñeca.

En pacientes con varianza cubital negativa, el objetivo es nivelar la articulación radiocubital distal a neutra o aproximadamente $1 \mathrm{~mm}$ de varianza cubital. Esto se puede lograr acortando el radio o alargando el cúbito. El alargamiento cubital requiere injerto óseo, y la posibilidad de falta de consolidación es mayor; por lo tanto, no se realiza comúnmente. , $^{8,9}$

Las osteotomías de acortamiento del radio son técnicamente simples y tienen una baja incidencia de falta de consolidación. Varios autores han publicado estudios de seguimiento a largo plazo que detallan los resultados de este procedimiento. Raven y cols. controlaron a 12 pacientes tratados, de esta manera, por un promedio de 22 años..$^{25}$ Uno tenía enfermedad en etapa 3B, y el resto, en etapa 2 o 3A. Observaron una progresión leve del cuadro en solo tres pacientes, en el último seguimiento, y el alivio del dolor y la función fueron excelentes en la mayoría. En un estudio similar, Watanabe y cols. evaluaron a 12 pacientes sometidos a osteotomía de acortamiento del radio en un seguimiento medio de 21 años. ${ }^{26}$ La mayoría tenía dolor leve en la muñeca, y mantuvo un buen rango de movilidad y función; sin embargo, las radiografías revelaron que la enfermedad había avanzado en el 50\% de los casos.

La osteotomía de acortamiento del radio en pacientes con estadio 3B sigue siendo controvertida. Altay y cols. compararon la eficacia del procedimiento en pacientes con estadios $3 \mathrm{~A}(\mathrm{n}=13)$ y $3 \mathrm{~B}(\mathrm{n}=10)$ en un seguimiento promedio de 85 meses. ${ }^{27}$ No hallaron diferencias significativas respecto al dolor y al rango de movilidad entre los diferentes estadios, y concluyeron en que este procedimiento obtiene resultados clínicamente similares, al margen del estadio, a pesar de la falta de mejoría radiológica.

Las opciones de nivelación articular para pacientes con varianza cubital neutra o positiva incluyen osteotomía en cuña de cierre de radio, osteotomía en domo del radio y osteotomía de acortamiento del hueso grande con artrodesis grande-ganchoso o sin ella. ${ }^{28}$ Las osteotomías radiales se utilizan para reducir la inclinación radial del segmento articular en un esfuerzo por disminuir las presiones de contacto entre el radio y el semilunar. Esta se logra aumentando el área de contacto entre el radio y el semilunar, y disminuyendo la fuerza que pasa a través de la articulación entre el radio y el semilunar y la articulación lunogrande. ${ }^{28}$ Koh y cols. comunicaron mejorías en el dolor, el movimiento y la función con un seguimiento mínimo de 10 años. ${ }^{29}$ Sin embargo, se observaron cambios artrósicos en casi las tres cuartas partes de los pacientes, en el último seguimiento, lo que sugiere que la enfermedad siguió avanzando. El objetivo de estos procedimientos es descargar las superficies articulares del semilunar mediante el desplazamiento de las cargas a las articulaciones adyacentes. En general, proporcionan resultados similares a los de la osteotomía de acortamiento del radio, con un buen alivio del dolor, restauración de la movilidad y la función, y posiblemente deteniendo o ralentizando el avance de la enfermedad.

La descompresión ósea metafisaria del radio es un concepto novedoso dirigido a pacientes con estadios de 1 a 3A. En la descripción original, Illarramendi y cols. reportaron su experiencia con este procedimiento en 22 pacientes, con un seguimiento promedio de 10 años. ${ }^{30}$ Este procedimiento consiste en descomprimir la metáfisis distal del radio a través de pequeñas ventanas corticales, utilizando una cureta u otro instrumento romo para impactar la esponjosa contra el canal medular, dejando las corticales sin esponjosa en los $3 \mathrm{~cm}$ distales del radio (Figura 1). 


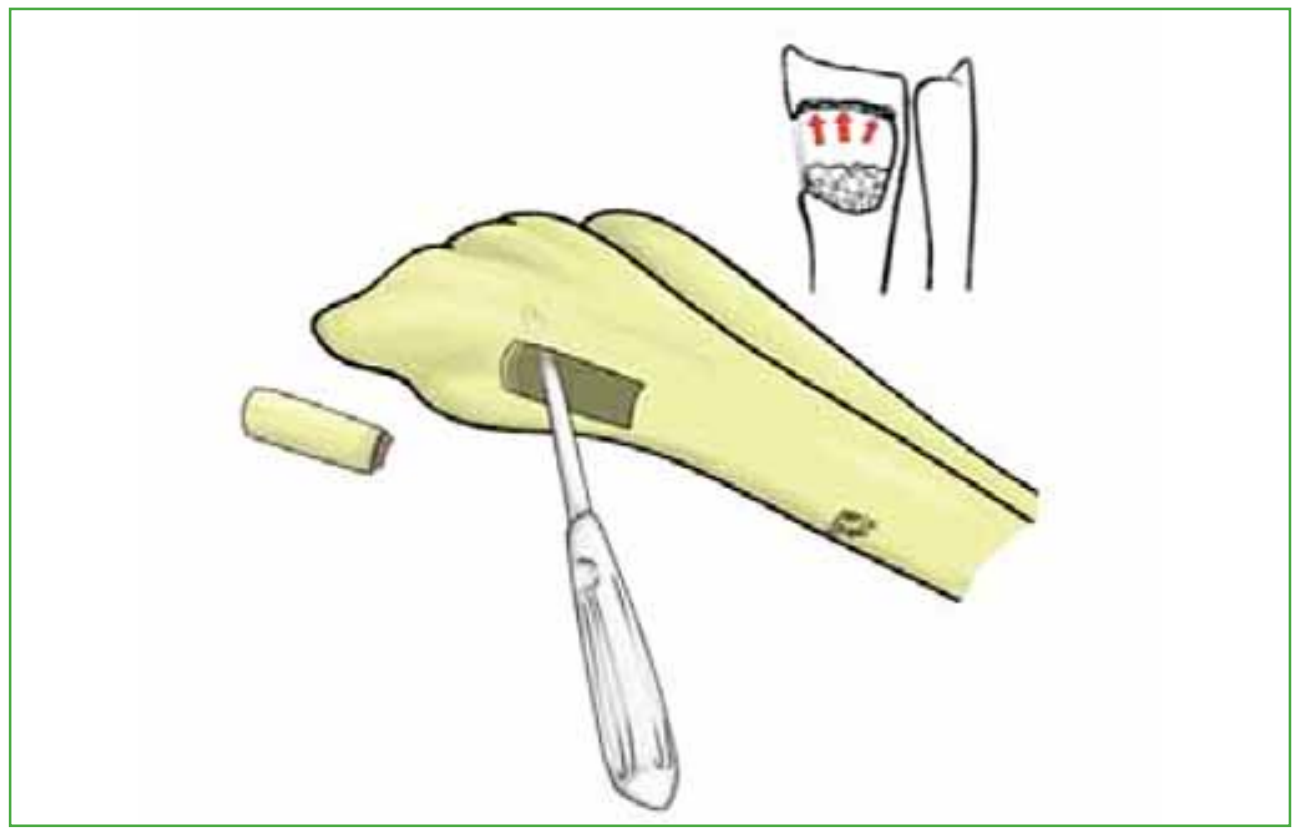

Figura 1. Ventana lateral por la cual se realiza la descompresión metafisaria del radio.

Los autores comunicaron una buena restauración del movimiento y alivio del dolor, solo cuatro pacientes refirieron dolor leve. En la mayoría (17 de 22 casos), la enfermedad no había avanzado, en el último seguimiento; se observó mejoría en las radiografías de dos y progresión en tres. En 2017, De Carli y cols. publicaron una serie de 16 pacientes con enfermedad de Kienböck en estadio 3A y un seguimiento promedio de 13 años; los resultados fueron excelentes en seis casos, buenos en ocho y pobres en uno, según el Mayo Wrist Score (Figura 2). ${ }^{31}$
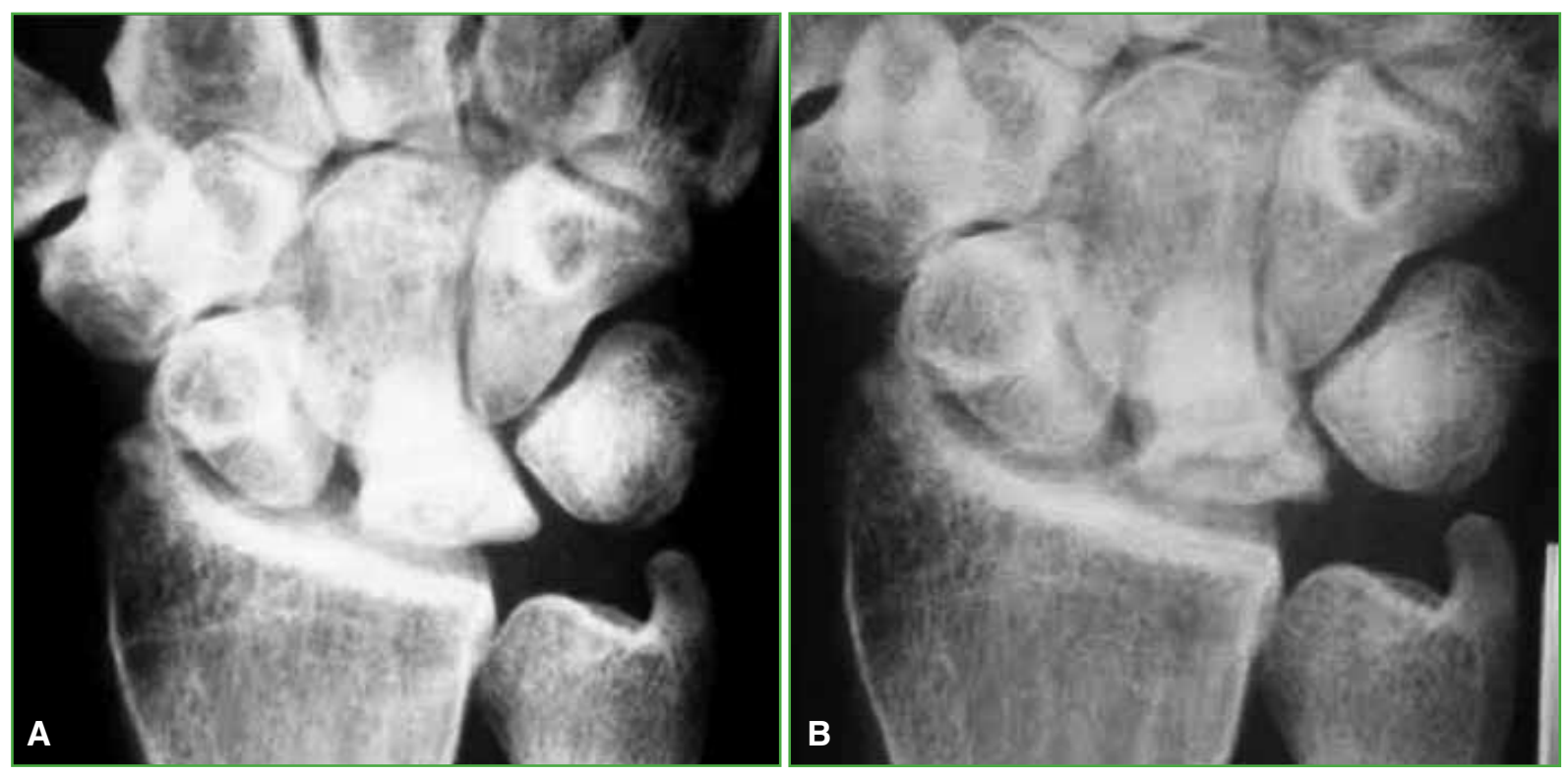

Figura 2. Mujer de 45 años con enfermedad de Kienböck en estadio 3A. A. Radiografía inicial. B. Control radiográfico a los 12 años de seguimiento. 


\section{Estadio 3B}

En esta etapa, hay pérdida de la altura del carpo debido a la flexión del escafoides, así como un colapso de la superficie articular semilunar. La corrección de la flexión del escafoides junto con la artrodesis intercarpiana (escafotrapeciotrapezoidal o escafogrande) puede disminuir la carga en el semilunar, lo que ayuda a estabilizar la articulación mediocarpiana y puede prevenir un mayor colapso carpiano. Además de la artrodesis, el semilunar puede ser removido y remplazado con tendón o un implante del semilunar para disminuir la irritación articular. Van den Dungen y cols. compararon los resultados de los pacientes tratados de forma conservadora $(n=19)$ y con artrodesis escafotrapeciotrapezoidal $(\mathrm{n}=11)$ durante un seguimiento medio de 13 años. Los autores afirman que los dos grupos fueron estadísticamente comparables y que la artrodesis escafotrapeciotrapezoidal produjo una mayor pérdida de la movilidad y dolor, y la rehabilitación fue más prolongada que con el tratamiento conservador. ${ }^{32}$ Tambe y cols. compararon los resultados de pacientes tratados con artrodesis intercarpiana parcial y artrodesis radiocarpiana para la enfermedad de Kienböck avanzada, y observaron que, en el último seguimiento, aquellos sometidos a artrodesis radiocarpiana tenían mejores resultados..$^{33}$

La carpectomía de fila proximal (CFP) es un procedimiento de rescate exitoso, pero es necesario que la cabeza del hueso grande no tenga cambios degenerativos. Si hay cambios artríticos leves en la cabeza del hueso grande, puede ser eficaz recurrir a una artroplastia por interposición utilizando la cápsula dorsal de la muñeca en conjunto con la CFP. ${ }^{34}$

Varios estudios comunican resultados detallados a largo plazo de la CFP para tratar la enfermedad de Kienböck. Croog y Stern publicaron una serie de 21 pacientes tratados por enfermedad en estadios 3 y 4 , y un seguimiento promedio de 10 años. ${ }^{35}$ Tres requirieron artrodesis radiogrande debido al dolor persistente, y dos de estos tres tenían enfermedad en estadio 4 en el momento del procedimiento inicial. Los autores concluyeron en que la CFP es un procedimiento confiable en esta situación, pero que se debe tener precaución al realizarlo en pacientes con enfermedad avanzada. En un estudio similar, DiDonna y cols. detallaron su experiencia con la CFP y un seguimiento mínimo de 10 años para pacientes con afecciones degenerativas de la muñeca, incluida la enfermedad de Kienböck. ${ }^{36}$ Nuevamente, los autores concluyeron en que la CFP es un procedimiento confiable en esta situación, aunque se debe tener precaución en pacientes $<35$ años. De Smet y cols. informaron sobre 21 pacientes con enfermedad de Kienböck avanzada con un seguimiento medio de 67 meses. ${ }^{37}$ Finalmente, Lumsden y cols. reportaron 13 pacientes con un promedio de 15 años de seguimiento. ${ }^{38} \mathrm{Al}$ igual que los autores anteriores, sostienen que es un procedimiento confiable a largo plazo para tratar la enfermedad de Kienböck avanzada.

\section{Estadio 4}

Los pilares del tratamiento para esta etapa incluyen la CFP y la artrodesis de muñeca. Como ya se mencionó, los cambios artríticos leves en la cabeza del hueso grande se pueden abordar en el momento de la CFP mediante una artroplastia por interposición de la cápsula dorsal de la muñeca. Sin embargo, es probable que los cambios artríticos en esta región generen la persistencia del dolor y el fracaso del procedimiento, la necesidad de una nueva intervención, la artrodesis. ${ }^{35}$

La desnervación de la muñeca sola o en combinación con los procedimientos antes mencionados puede ayudar a aliviar los síntomas. Schweizer y cols. publicaron su experiencia a largo plazo con la desnervación completa de la muñeca en una serie de 70 pacientes, 11 de ellos tenían enfermedad de Kienböck. ${ }^{39}$ El seguimiento promedio fue de 9.6 años. La desnervación completa de la muñeca condujo a una mejora subjetiva en aproximadamente dos tercios de los pacientes, y alrededor de la mitad refirió la desaparición completa o marcada del dolor.

La enfermedad de Kienböck sigue siendo un desafío clínico para los cirujanos de mano. Los factores etiológicos detrás del desarrollo de la enfermedad permanecen sin identificar. Los avances quirúrgicos recientes en la forma de injertos óseos vascularizados del radio distal tienen el potencial para mejorar el tratamiento de esta afección. Los procedimientos tradicionales para tratar la enfermedad avanzada, como la osteotomía de acortamiento de radio y la CFP, han logrado un adecuado control de los síntomas y la restauración de la función en estudios a largo plazo.

Conflicto de intereses: Los autores no declaran conflictos de intereses. 


\section{BIBLIOGRAFÍA}

1. Wagner JP, Chung KC. A historical report on Robert Kienböck (1871-1953) and Kienböck's disease. J Hand Surg Am 2005;30:1117-21. https://doi.org/10.1016/j.jhsa.2005.08.002

2. Hultén O. Uber anatomische variationen der handgelenkknochen. Acta Radiol Scand 1928;9:155-68. https://doi.org/10.3109/00016922809176658

3. Nakamura R, Tsuge S, Watanabe K, Tsunoda K. Radial wedge osteotomy for Kienböck's disease. J Bone Joint Surg Am 1991;73:1391-6. PMID: 1918122

4. Gelberman RH, Bauman TD, Menon J, Akeson WH. The vascularity of the lunate bone and Kienböck's disease. J Hand Surg Am 1980;5(3):272-8. https://doi.org/10.1016/s0363-5023(80)80013-x

5. Panagis JS, Gelberman RH, Taleisnik J, Baumgaertner M. The arterial anatomy of the human carpus. Part II: the intraosseous vascularity. J Hand Surg Am 1983;8(4):375-82. https://doi.org/10.1016/s0363-5023(83)80195-6

6. Allan CH, Joshi A, Lichtman DM. Kienbock's disease: diagnosis and treatment. J Am Acad Orthop Surg 2001;9:128-36. https://doi.org/10.5435/00124635-200103000-00006

7. Pichler M, Putz R. The venous drainage of the lunate bone. Surg Radiol Anat 2003;24:372-6. https://doi.org/10.1007/s00276-002-0065-y

8. Luo J, Diao E. Kienböck's disease: an approach to treatment. Hand Clin 2006;22:465-73. https://doi.org/10.1016/j.hcl.2006.07.003

9. Schuind F, Eslami S, Ledoux P. Kienböck's disease. J Bone Joint Surg Br 2008;90:133-9. https://doi.org/10.1302/0301-620X.90B2.20112

10. Bain GI, Begg M. Arthroscopic assessment and classification of Kienbock's disease. Tech Hand Up Extrem Surg 2006;10:8-13. https://doi.org/10.1097/00130911-200603000-00003

11. Goldfarb CA, Hsu J, Gelberman RH, Boyer MI. The Lichtman classification for Kienböck's disease: an assessment of reliability. J Hand Surg Am 2003;28:74-80. https://doi.org/ https://doi.org/10.1053/jhsu.2003.50035

12. Salmon J, Stanley JK, Trail IA. Kienböck's disease: conservative management versus radial shortening. J Bone Joint Surg Br 2000;82:820-3. https://doi.org/10.1302/0301-620x.82b6.10570

13. Beckenbaugh RD, Shives TC, Dobyns JH, Linscheid RL. Kienböck's disease: the natural history of Kienböck's disease and considerations of lunate fractures. Clin Orthop 1980;149:98-106. PMID: 7408323

14. Keith PP, Nuttall D, Trail I. Long-term outcome of nonsurgically managed Kienböck's disease. J Hand Surg Am 2004;29:63-7. https://doi.org/10.1016/j.jhsa.2003.10.016

15. Delaere O, Dury M, Molderez A, Foucher G. Conservative versus operative treatment for Kienböck's disease: a retrospective study. J Hand Surg Br 1998;23:33-6. https://doi.org/10.1016/s0266-7681(98)80214-3

16. Lichtman DM, Roure AR. External fixation for the treatment of Kienböck's disease. Hand Clin 1993;9:691-7. PMID: 8300738

17. Moran SL, Shin AY. Vascularized bone grafting for the treatment of carpal pathology. Orthop Clin North Am 2007;38:73-85. https://doi.org/10.1016/j.ocl.2006.10.010

18. Daecke W, Lorenz S, Wieloch P, Jung M, Martini AK. Vascularized os pisiform for reinforcement of the lunate in Kienböck's disease: an average of 12 years of follow-up study. J Hand Surg Am 2005;30:915-22. https://doi.org/10.1016/j.jhsa.2005.03.019

19. Sheetz KK, Bishop AT, Berger RA. The arterial blood supply of the distal radius and ulna and its potential use in vascularized pedicled bone grafts. J Hand Surg Am 1995;20:902-14. https://doi.org/10.1016/S0363-5023(05)80136-4

20. Bochud RC, Büchler U. Kienböck's disease, early stage 3: height reconstruction and core revascularization of the lunate. J Hand Surg Br 1994;19:466-78. https://doi.org/10.1016/0266-7681(94)90212-7

21. Tamai S, Yajima H, Ono H. Revascularization procedures in the treatment of Kienböck's disease. Hand Clin 1993;9:455-66. PMID: 8408255

22. Moran SL, Cooney WP, Berger RA, Bishop AT, Shin AY. The use of the 4-5 extensor compartmental vascularized bone graft for the treatment of Kienbock's disease. J Hand Surg Am 2005;30:50-8. https://doi.org/10.1016/j.jhsa.2004.10.002

23. Gabl M, Lutz M, Reinhart C, Zimmerman R, Pechlaner S, Hussl H, et al. Stage 3 Kienböck's disease: reconstruction of the fractured lunate using a free vascularized iliac bone graft and external fixation. J Hand Surg Br 2002;27:36973. https://doi.org/10.1054/jhsb.2002.0766 
24. Arora R, Lutz M, Deml C, Krappinger D, Zimmermann R, Gabl M. Long-term subjective and radiological outcome after reconstruction of Kienböck's disease stage 3 treated by a free vascularized iliac bone graft. J Hand Surg Am 2008;33:175-81. https://doi.org/10.1016/j.jhsa.2007.11.005

25. Raven EE, Haverkamp D, Marti RK. Outcome of Kienböck's disease 22 years after distal radius shortening osteotomy. Clin Orthop Relat Res 2007;460:137-41. https://doi.org/10.1097/BLO.0b013e318041d309

26. Watanabe T, Takahara M, Tsuchida H, Yamahara S, Kikuchi N, Ogino T. Long-term follow-up of radial shortening osteotomy for Kienböck disease. J Bone Joint Surg Am 2008;90(8):1705-11. https://doi.org/10.2106/JBJS.G.00421

27. Altay T, Kaya A, Karapinar L, Ozturk H, Kayali C. Is radial shortening useful for Litchman stage 3B Kienbock's disease? Int Orthop 2008;32(6):747-52. https://doi.org/10.1007/s00264-007-0428-4

28. Watanabe K, Nakamura R, Horii E, Miura T. Biomechanical analysis of radial wedge osteotomy for the treatment of Kienböck's disease. J Hand Surg Am 1993;18:686-90. https://doi.org/10.1016/0363-5023(93)90319-X

29. Koh S, Nakamura R, Horii E, Nakao E, Inagaki H, Yajima H. Surgical outcome of radial osteotomy for Kienböck's disease-minimum 10 years of follow-up. J Hand Surg Am 2003;28:910-6. https://doi.org/10.1016/s0363-5023(03)00490-8

30. Illarramendi AA, Schulz C, De Carli P. The surgical treatment of Kienböck's disease by radius and ulna metaphyseal core decompression. J Hand Surg Am 2001;26:252-60. https://doi.org/10.1053/jhsu.2001.22928

31. De Carli P, Zaidenberg EE, Alfie V, Donndorff A, Boretto JG, Gallucci GL. Radius core decompression for Kienböck disease stage IIIA: outcomes at 13 years follow-up. J Hand Surg Am 2017;42(9):752.e1-752.e6. https://doi.org/10.1016/j.jhsa.2017.05.017

32. Van den Dungen S, Dury M, Foucher G, Marin Braun F, Loréa P. Conservative treatment versus scaphotrapeziotrapezoid arthrodesis for Kienböck's disease. A retrospective study. Chir Main 2006;25:141-5. https://doi.org/10.1016/j.main.2006.07.030

33. Tambe AD, Trail IA, Stanley JK. Wrist fusion versus limited carpal fusion in advanced Kienbock's disease. Int Orthop 2005;29:355-8. https://doi.org/10.1007/s00264-005-0013-7

34. Salomon GD, Eaton RG. Proximal row carpectomy with partial capitate resection. J Hand Surg Am 1996;21:2-8. https://doi.org/10.1016/S0363-5023(96)80147-X

35. Croog AS, Stern PJ. Proximal row carpectomy for advanced Kienböck's disease: average 10-year follow-up. $J$ Hand Surg Am 2008;33:1122-30. https://doi.org/10.1016/j.jhsa.2008.02.031

36. DiDonna ML, Kiefhaber TR, Stern PJ. Proximal row carpectomy: study with a minimum of ten years of follow-up. J Bone Joint Surg Am 2004;86:2359-65. PMID: 15523004

37. De Smet L, Robijns P, Degreef I. Proximal row carpectomy in advanced Kienböck's disease. J Hand Surg Br 2005;30:585-7. https://doi.org/10.1016/j.jhsb.2005.06.024

38. Lumsden BC, Stone A, Engber WD. Treatment of advanced-stage Kienböck's disease with proximal row carpectomy: an average 15- year follow-up. J Hand Surg Am 2008;33:493-502. https://doi.org/10.1016/j.jhsa.2007.12.010

39. Schweizer A, von Känel O, Kammer E, Meuli-Simmen C. Long-term follow-up evaluation of denervation of the wrist. J Hand Surg Am 2006;31:559-64. https://doi.org/10.1016/j.jhsa.2005.12.012 